\title{
Landslide susceptibility mapping using AHP method and GIS in the peninsula of Tangier (Rif-northern morocco)
}

\author{
Ait Brahim L, Bousta M, Jemmah I A, El Hamdouni I, ElMahsani A, Abdelouafi A,. Sossey alaoui F. and Lallout I \\ Université Mohammed V, Faculté des Sciences, Département sciences de la terre, Unité de recherche GEORISK, LGRN, \\ 4 Avenue Ibn Battouta Rabat-Agdal, BP 1014, Rabat, Maroc.
}

\begin{abstract}
The peninsula of Tangier (Northern Morocco) is submitted to a significant number of landslides each year due to its lithological, structural and morphological complexity; which cause a lot of damage to the road network and other related infrastructure. The main objective of this study is to create a landslide indexed susceptibility map of Tangier peninsula, by using AHP ( Analytical Hierarchical Processes) model to calculate each factor's weight. The work is made via GIS by using an ArcGIS AHP extension. In the current research, First of all, the four main types of landslides were identified and mapped from existing documents, works and new data which came from either remote sensing or fieldwork. Lithology, land use, slope, hypsometry, exposure, fault density and drainage network density were used as main parameters controlling the occurrence of the selected landslides. Then, afterward, each parameter is classified into a number of significant classes based on their relative influence on gravitational movement genesis. The validity of the susceptibility zoning map which is obtained through linear summation of indexed maps was tested and cross-checked by inventoried and studied landslides. The obtained landslide susceptibility map constitutes a powerful decision-making tool in land-use planning, i.e. New highways, secondary highways, railways, etc. within the national development program in the Northern provinces. It is a necessary step for the landslides hazard assessment in the Tangier peninsula in northern Morocco.
\end{abstract}

Key words: Landslides, remote sensing, indexed parameters, susceptibility map, land-use planning, peninsula of Tangier, Rif, Morocco.

\section{Introduction}

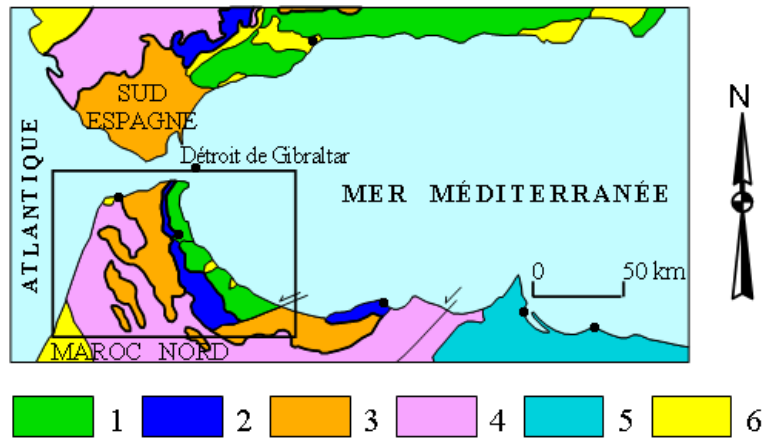

Fig. 1. Secteur d'étude : $1:$ domaine d'Alboran, $2:$ dorsale calcaire, 3 : nappes de flysch, $4:$ intrarif, $5:$ avant pays, 6 : dépôt post nappes.

La chaîne du Rif et plus particulièrement la péninsule de Tanger (figure 1), présente la fréquence la plus importante, au Maroc, en termes d'instabilités de versants du fait de sa complexité géologique, morphologique et climatique [1-8]. Ces instabilités entraînent chaque année des dégâts en vies humaines, au niveau des habitations, des aménagements hydrauliques, électriques, des terres agricoles, du réseau ferroviaire et surtout le réseau routier. Actuellement, les villes de la péninsule de Tanger (Tétouan, Chefchaouen, Tanger, etc.) subissent une pression démographique (exode rural). En effet, le périmètre urbain bâti a déjà comblé toutes surfaces aménageables stables et facilement constructibles au niveau de ses trois villes. La croissance urbaine a entraîné et entraînera le débordement de l'urbanisme sur de nouveaux versants et plateaux adjacents. Le démarrage du plan d'aménagement des provinces du nord dont le but, est le désenclavement de cette région, a nécessité actuellement le lancement de constructions de nouveaux barrages (Martil), unités de productions d'énergie (centrales électriques, éoliennes) et d'infrastructures portuaires (MED1, MED2 etc.). Il a surtout permis la mise en place d'un important réseau de communication (autoroutes, rocade méditerranéenne, chemin de fer, lignes grande vitesse, aéroports, routes nationales, secondaires, etc.). Tous ses grands projets nécessitent l'établissement d'une nouvelle cartographie pour mettre en évidence les zones les plus exposées aux instabilités de terrain. L'objectif principal de cette étude est de cartographier la susceptibilité des mouvements de masse en utilisant l'AHP (approche heuristique) [9], [21]. 


\section{Matériels et méthodologie}

Dans le cadre de cette étude, nous avons eu recours aux cartes géologiques 1/100 000 et topographiques 1/50 $000 \mathrm{du}$ Rif septentrional; aux données satellitaires Landsat7 ETM+ ( résolution 30m, Panchro 15m), Spot 5 THR (Très haute résolution spatiale de 2,5 m, Google Earth professionnel), Radar ERS-1(résolution $12.5 \mathrm{~m}$ ). Les données des précipitations des stations météorologiques du Rif (1941-2016; DGH, 2017). Les données de l'occupation des sols en termes d'essences forestières (HCEFLCD, 2016). Les images satellitaires ont été fournies dans le cadre de l'action intégrée (AI 219 STU) entre la Faculté des Sciences de Rabat et l'Université Pierre et Marie Curie (Paris VI).

L'approche adoptée lors de cette étude comprend trois étapes principales: l'acquisition de l'information, l'élaboration d'une base de données numérique à l'aide d'un SIG et la zonation de la susceptibilité à échelle régionale et moyenne (méso-échelle)

1. La première étape, a été l'acquisition de l'information: Inventaire, caractérisation et cartographie des mouvements de masses à partir de cartes thématiques, de photographies aériennes, d'images satellitaires, d'identification et de contrôle sur le terrain.

2. Elaboration d'une base de données multisources et multi-échelles des facteurs de prédisposition de l'occurrence spatiale des mouvements de terrain, sélectionnés de part leur disponibilité et leur pertinence (variable à modéliser) ; Cette base de données a nécessité la consultation et la synthèse des données bibliographiques du secteur d'étude, l'utilisation de l'imagerie satellitaires disponibles (cf supra) et l'identification et le contrôle sur le terrain dans la mesure du possible.

3. Élaboration de la carte de susceptibilité des mouvements de masse à l'échelle régionale en combinant des cartes d'indices [21] après l'utilisation de l'approche AHP pour l'organisation des différents paramètres et l'attribution de poids relatifs à chaque classe [12].

\subsection{Cartographie des mouvements de terrain: typologie, classification et inventaire}

Les données extraites des images satellitaires associées à nos observations de terrain ont été d'un apport important pour la cartographie et l'inventaire des mouvements de masse de la péninsule de Tanger. La carte numérique géoréférencée de synthèse de la localisation et de la distribution spatiale des mouvements de terrains que nous avons élaborés présente quatre grands classes (Figure2) suivant les traits morphologiques les plus dominants: les écroulements (2.5\%), les glissements $(11.7 \%)$, les coulées boueuses $(19.4 \%)$ et les glissements complexes $(66.4 \%)$. Les mouvements sont représentés en tenant compte de l'échelle par des polygones qui délimitent les surfaces affectées.
Cependant, pour plus de lisibilité des cartes présentées dans le présent travail, les 4 classes ont été représentées par des points de formes et de couleurs différentes. Cette synthèse a permis d'élaborer une carte de densité des mouvements de terrain qui correspond à une zonation préliminaire (hiérarchisée par des courbes d'isovaleurs) d'où se dégage les secteurs de plus grande occurrence. Cette carte va permettre aussi, de quantifier l'abondance spatiale des mouvements inventoriés en termes de fréquences. Le croisement de la densité des mouvements de terrain de la péninsule de Tanger avec les différents facteurs individuellement, permet à ce stade d'avoir une idée même globale sur le degré d'importance de chaque paramètre dans l'occurrence des mouvements de terrain. Cette carte servira de base pour tester le degré de validité de la carte de susceptibilité obtenue.

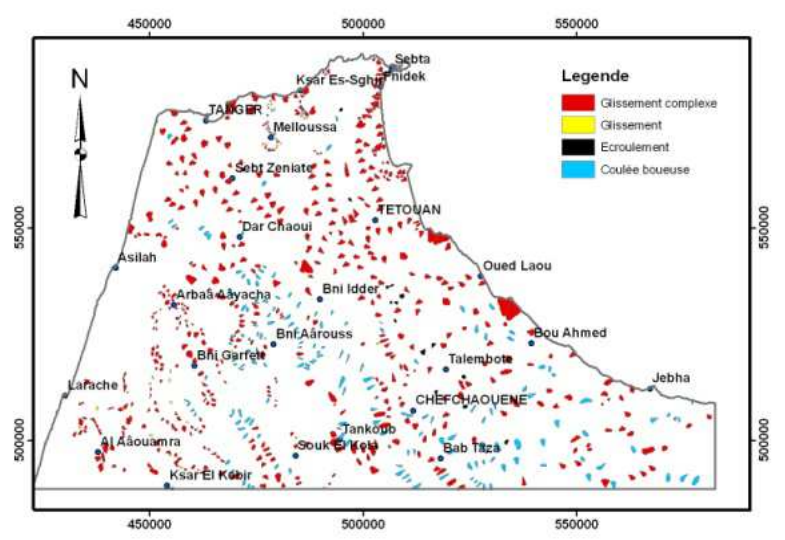

Fig. 2. Carte d'inventaire des instabilités de terrain

\subsubsection{Facteurs de prédisposition}

L'identification et la spatialisation des variables prédictives (paramètres de prédisposition) des mouvements de terrain de la péninsule de Tanger a nécessité la consultation et la synthèse des données bibliographiques disponibles, l'utilisation de l'imagerie satellitaires et des missions sur le terrain. La base de données multi-sources et multi-échelles des paramètres de prédisposition (lithologie, occupation de sol, fracturation, hypsométrie, pente, exposition) élaborée va permettre le croisement et la superposition entre eux et avec la variable à modéliser les mouvements de terrain, ainsi que leur mise à jour en fonction des nouvelles données acquises. Elle servira surtout à l'élaboration de la carte de susceptibilité.

\subsubsection{Lithologie}

L'occurrence et la distribution spatiale des glissements que nous avons identifiés et cartographiés montre qu'un nombre important de mouvements de masses se développent sur des affleurements de faciès assez diversifié. Pour l'extraction de l'information lithologique à l'échelle régionale, on a eu recourt à la partie couverte par les cartes géologiques $1 / 100000$ et $1 / 50000$ et le reste à partir de la carte géologique du Rif 1/500 000ème et les données satellitaires Landsat7 ETM+ et Spot 5 THR (Google Earth professionnel) grâce à plusieurs rehaussements spectraux (l'indice lithologique de Bardinet[17]; la composition colorée minéralogique 
avec le triplet de Jenssen[18], l'ACP etc.). La carte de faciès élaborée (Figure3) montre une variation lithologique des affleurements que l'on peut regrouper en six grandes classes :1-marnes-argils, 2-grès, 3-dolomiescalcaires, 4-calcaires marneux, 5: les alluvions, 6-grès marneux. Les faciès marneux (l'unité de Tanger et de Beni Ider) sont caractérisés par l'aspect mécanique médiocre des argiles (riches en smectite) et leur sensibilité aux variations de la teneur en eau [13], [7] .

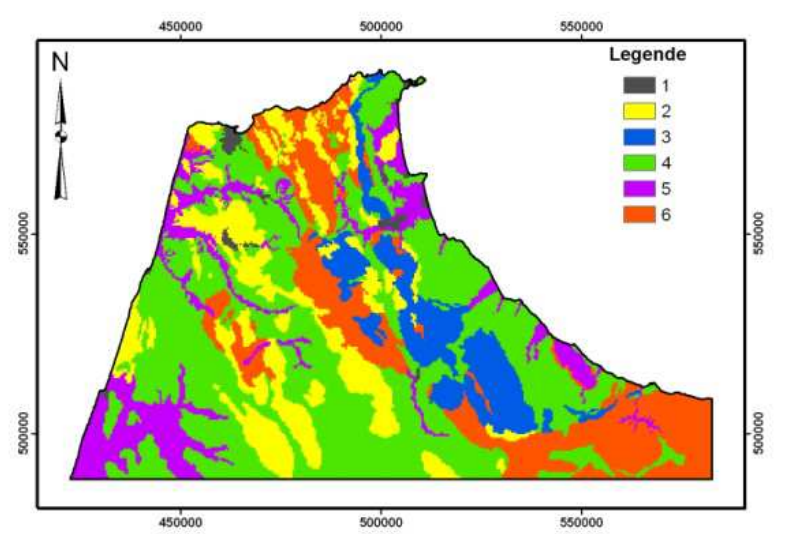

Fig. 3. Carte lithologique

\subsubsection{Hypsométrie}

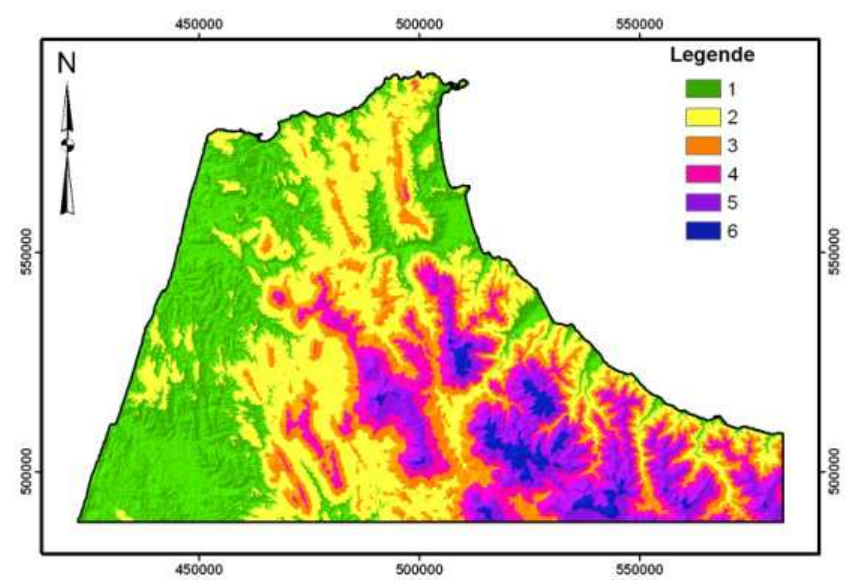

Fig. 4. Carte hypsométrique

La carte d'hypsométrie a été dérivée du MNT GDEM Aster 30m. Les altitudes varient entre le "zéro" (nivellement général marocain) et $2159 \mathrm{~m}$ point culminant du (Jbel Tissouka), ce qui a conditionné le choix de l'équidistance des courbes de niveaux pour mettre en relief l'ampleur des phénomènes morphologiques. La superposition de la carte des mouvements de terrain cartographiés à la carte hypsométrique montre que d'une façon générale: plus l'altitude augmente, plus la fréquence d'occurrence de mouvement de terrain est présente. Cependant, nous avons remarqué que certains affleurements de roches carbonatées massifs (à $1926 \mathrm{~m}$ et $2159 \mathrm{~m}$ ), et de grès $(1658 \mathrm{~m})$ sont le siège de ruptures par écroulements et coulées de débris mais avec des taux inférieurs à la moyenne générale. Ainsi, le relief, par sa composante altimétrique constitue l'un des facteurs les plus déterminants dans la genèse des glissements de terrains de par sa relation directe avec la répartition de la pluviométrie (moyenne annuelle oscille entre $500 \mathrm{~mm}$ en basse altitude à plus $1200 \mathrm{~mm}$ sur les crêtes). Nous avons défini six classes d'altitudes (1:0-100 m; $2: 100-300 \mathrm{~m}$; 3 :300-400m ; 4 :400-600m ;5 :600-1200m ; 6 : >1200m).

\subsubsection{Pente}

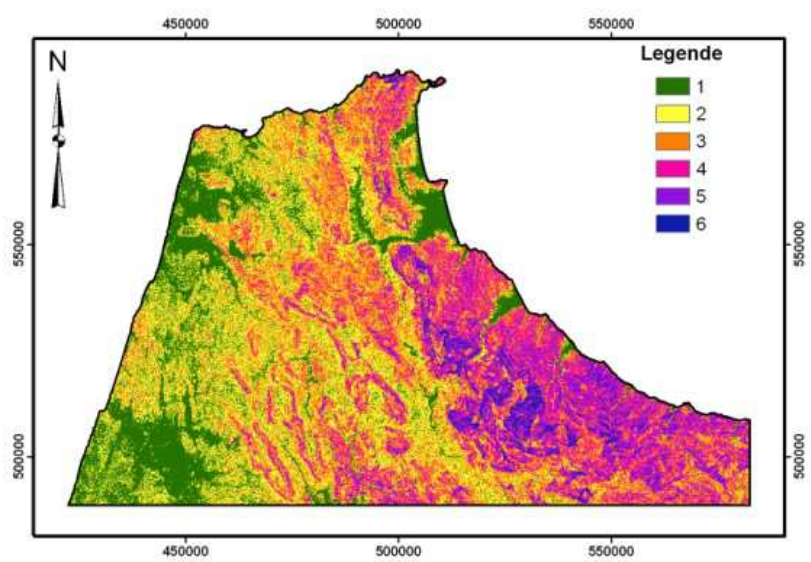

Fig. 5. Carte des pentes

Lors de la cartographie des glissements dans la péninsule de Tanger, les versants les plus propices à l'instabilité sont ceux dont l'inclinaison dépasse $5^{\circ}$, avec un maximum de susceptibilité entre $10^{\circ}$ et $35^{\circ}$. Par typologie, les versants montrent la même tendance de comportement vis-à-vis de l'instabilité due aux mouvements complexes et aux coulées, mais avec des valeurs différentes. Le taux de rupture par glissement s'accroît avec l'inclinaison des versants. Sous un degré d'instabilité relativement faible, les pentes très fortes paraissent les plus sensibles aux éboulements et aux écroulements. Ainsi, pour le calcul de l'aléa des instabilités de versants, six classes $\left(1:\left(0^{\circ}-2.5^{\circ}\right) ; 2:\left(2.5^{\circ}\right.\right.$ $\left.5^{\circ}\right)$; 3: $\left(5^{\circ}-10^{\circ}\right) ; 4:\left(10^{\circ}-20^{\circ}\right) ; 5$ : $\left.\left(20^{\circ}-30^{\circ}\right) ; 6:\left(>30^{\circ}\right)\right)$ de pentes (Figure 5) dérivées du MNT correspondant à six indices d'instabilité allant de $5^{\circ}$ à $80^{\circ}$ ont été établies sur la base d'une étude statistique comparative entre la densité des surfaces occupées par le phénomène de glissement et les pentes.

\subsubsection{Exposition}

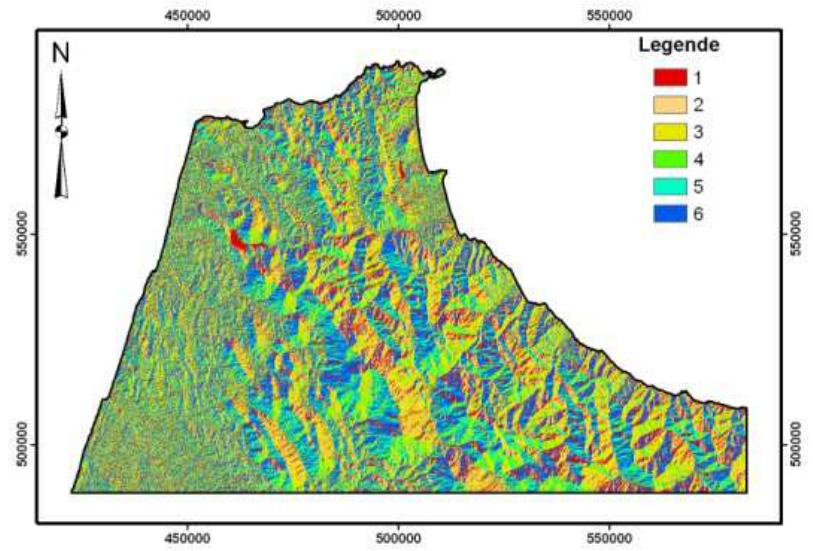

Fig. 6. Carte d'exposition

La carte d'exposition élaborée (Figure 6) montre 6 classes d'orientation des versants $(1: \mathrm{N} ; 2: \mathrm{NE} ; 3: \mathrm{E}$ à 
$\mathrm{SE} ; 4$ :S ; 5 :SW ; 6 : W à NW). A l'échelle régionale, les expositions E, W et NW, SE, sont largement dominantes. Mais, à l'échelle du versant, une relative homogénéité de distribution des terrains, selon leur exposition par rapport au nord, est mise en évidence. A l'exception des terrains plats couverts principalement par des alluvions récents.

\subsubsection{Fracturation}

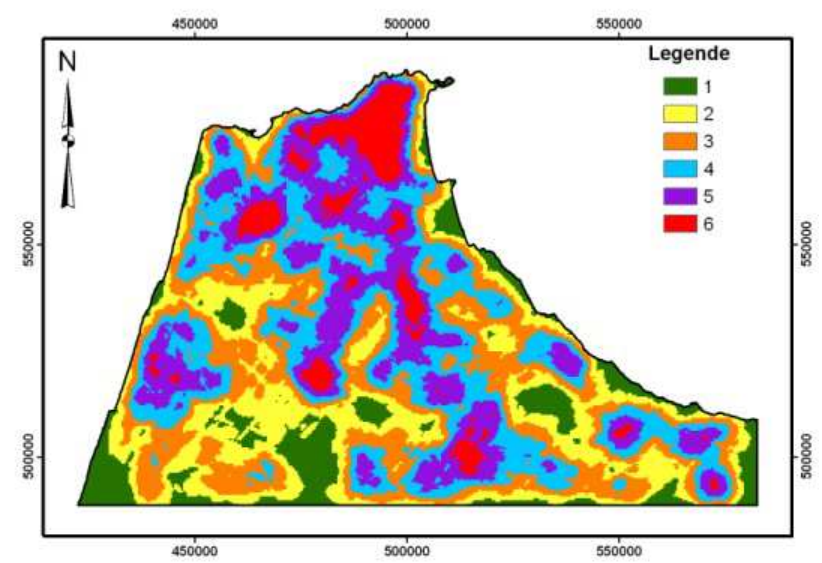

Fig. 7. Carte de densité de fracturation

La carte de synthèse de la fracturation de la péninsule de Tanger est le résultat de l'utilisation de 3 sources. La digitalisation des failles extraites des documents existants (cartes géologiques 1/50 000,1/100 000 et 1/500 000). La fracturation obtenue à partir de l'analyse de l'imagerie satellitaire Radar ERS1,Spot XS et Landsat7 ETM+[15] [24], et la carte de la fracturation récente obtenue à partir de l'analyse du réseau hydrographique en terme d'anomalie et de perturbation du réseau hydrographique. La carte synthétique de la fracturation s'organise selon 3 directions NE-SW, ENEWSW et NW-SE. Ceci nous a permis d'établir une carte de densité de la fracturation (Figure 7) qui tient compte aussi bien de la longueur de la faille que de la présence de nœuds tectoniques. Les travaux effectués sur la tectonique mio-pliocène et quaternaire de la péninsule de Tanger montrent l'importance de ces directions dans la poursuite de la structuration de cette dernière [15],[19],[20]. Ceci est attesté aussi par le fait que ses failles ont d'une part guidé le tracé du réseau hydrographique [23] et d'autre part montrent une relation avec la répartition de l'activité sismique dans la péninsule de Tanger [22]. Sur le terrain, on a remarqué que des mouvements de terrain se situent le long de certains failles, à proximité de certains nœuds tectoniques (NESW et NW-SE) et de zone de broyage des failles (cisaillements et/ou décrochements) formée de matériaux fragilisés et de résurgences. Ce paramètre nécessite l'introduction de la notion de distance des mouvements de terrain par rapport aux failles [5]. Un certain nombre de failles permettent à l'eau de s'infiltrer entraînant ainsi l'accroissement de pressions interstitielles et la réduction de la résistance au cisaillement des sols [15]. Une fois que ces derniers arrivent à saturation, le mouvement de masse est déclenché avec formation d'un plan de cisaillement profond.

\subsubsection{Occupations de sol}

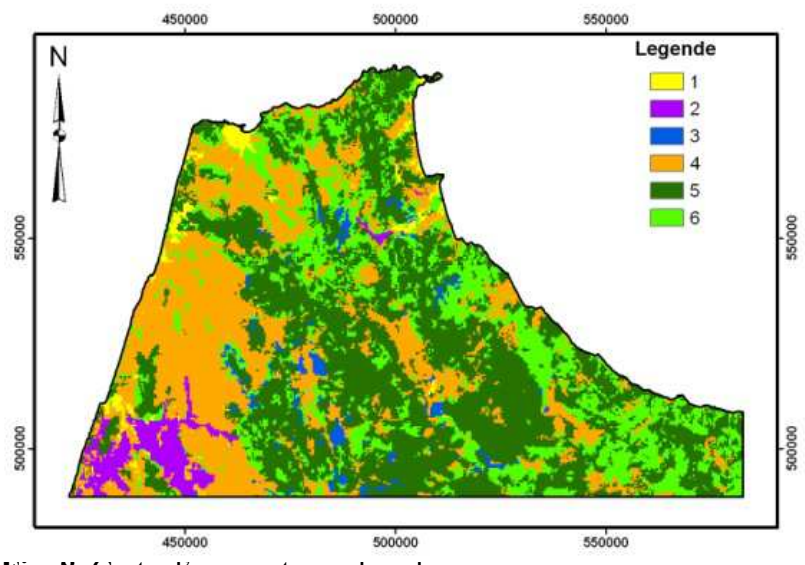

Fig. 8. Carte d occupations de sol

La couverture végétale de la péninsule de Tanger exerçait jusqu'au années 1960 une action phytostabilisante sur les versants à substrat argileux ou à substrat rocheux [1-3]. Cette action se manifeste par l'atténuation de l'énergie cinétique des eaux de ruissellement et des précipitations, l'évapotranspiration, l'amélioration de la qualité physique du sol par la structure des racines et surtout la fixation du manteau d'altération susceptible de glisser par rapport au substratum. Depuis 1970, l'augmentation de la fréquence des années de sécheresse, les feux de forêts et l'action anthropique par le défrichement et surtout le développement accéléré du réseau routier (routes nationales, secondaires, rocade méditerranéenne, chemin de fer, autoroutes, lignes grande vitesse, etc.) ont accentué les différentes formes d'érosion superficielles et profondes rendant ainsi le sol plus conditionné à l'occurrence des mouvements de masses. Pour la cartographie de l'occupation du sol, nous avons utilisé les données de l'occupation des sols en termes d'essences forestières fourni par le HCEFLCD (2016). L'actualisation des données a été effectuée par interprétation visuelle des données satellitaires Spost5 très haute résolution. Nous avons aussi appliqué des classifications supervisées par maximum de vraisemblance sur une mosaïque de données Landsat ETM+. Ensuite, une vérification de la carte obtenue, par un certain nombre de contrôles sur le terrain. La carte de l'occupations du sols (Figure 8) a été classifiée en 6 classes: 1: plans d'eau, bâti et espaces urbains - 2 : périmètres irrigués et terrains nus - 3 : reboisements 4 : terres arables -5 : forêts et végétation naturelle - 6 : terrains agricoles.

\subsubsection{Drainage}

Le réseau hydrographique a été digitalisé à partir des cartes topographiques 1/100 000. La carte de sa densité élaborée comporte six classes (Figure 9). L'action érosive du réseau hydrographique peut causer la déstabilisation du terrain et donc participer fortement à l'occurrence des glissements de terrain. Plusieurs instabilités de terrains cartographiées sont réactivées le 
long de certains oueds surtout lors des périodes de crues (plus particulièrement au niveau des méandres).

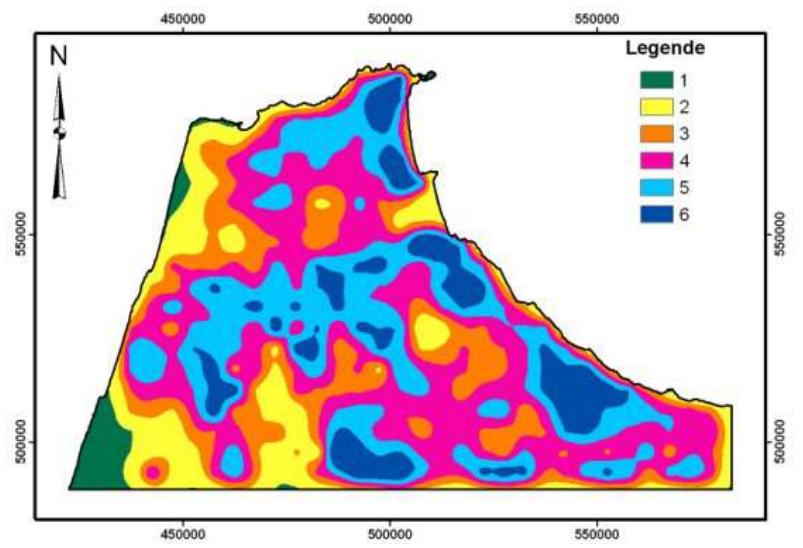

Fig. 9. Carte de densité de drainage

\section{Cartographie de la susceptibilité aux instabilités de terrain par l'approche AHP}

La cartographie de la susceptibilité aux instabilité de terrain par combinaison des cartes d'index adoptée dans le présent travail a été proposée initialement par Carrara et al., [9]. Il s'agit d'une méthode heuristique indirecte et qualitative pour l'évaluation de la susceptibilité aux mouvements de terrain. Elle a nécessité la hiérarchisation [12] des différents paramètres significatifs prédictifs sélectionnés (lithologie, fracturation, la pente, hypsometrie, exposition, occupation du sol). La valeur des poids relatifs attribuée est basée sur nos connaissances du contexte géodynamique responsable du développement des instabilités de terrain dans la péninsule de Tanger [14],[15] et[5]. Pour générer la matrice d'AHP, les six facteurs de prédisposition ont été utilisés dans une comparaison "pair-par-pair". Ces facteurs ont d'abord été standardisés et classés en six classes. Nous avons également assigné des poids pour chaque classe de paramètres prédictifs en fonction du pourcentage de glissements de terrain inventoriés présents dans cette classe [16]. Finalement, une seule et unique carte d'index de l'AMT (IAMT) a été élaborée à l'aide de la formule ci-dessous proposée par Voogd [21].

$$
I A_{M T}=\sum_{j=I}^{n} \boldsymbol{W}_{j} \boldsymbol{w}_{i j}
$$

Avec :

\footnotetext{
$I A_{M T}$ :Index de susceptibilité aux mouvements de terrain $W_{j}$ : Poids assigné au paramètre ' $\mathrm{j}$ '

$\mathcal{W}_{i j}$ : Poids assigné la classe ‘i’ paramètre ‘j c

$\mathrm{n}$ : Nombre de paramètres
}

Table 1. Matrice AHP des poids

\begin{tabular}{|c|c|c|c|c|c|c|c|}
\hline & Hyps & Lith & Occu & Expo & Pent & Frac & Hydr \\
\hline Hyps & 1 & 2 & 3 & 4 & 5 & 6 & 7 \\
\hline Lith & 0,5 & 1 & 1 & 3 & 4 & 5 & 6 \\
\hline Occu & 0,33 & 1 & 1 & 3 & 4 & 5 & 6 \\
\hline Expo & 0,25 & 0,33 & 0,33 & 1 & 1 & 2 & 3 \\
\hline Pente & 0,2 & 0,25 & 0,25 & 1 & 1 & 2 & 3 \\
\hline Frac & 0,17 & 0,2 & 0,2 & 0,5 & 0,5 & 1 & 2 \\
\hline Hydr & 0,14 & 0,17 & 0,17 & 0,33 & 0,33 & 0,5 & 1 \\
\hline Wi & 0,36 & 0,21 & 0,2 & 0,08 & 0,07 & 0,05 & 0,03 \\
\hline
\end{tabular}

\section{Résultats et conclusions}

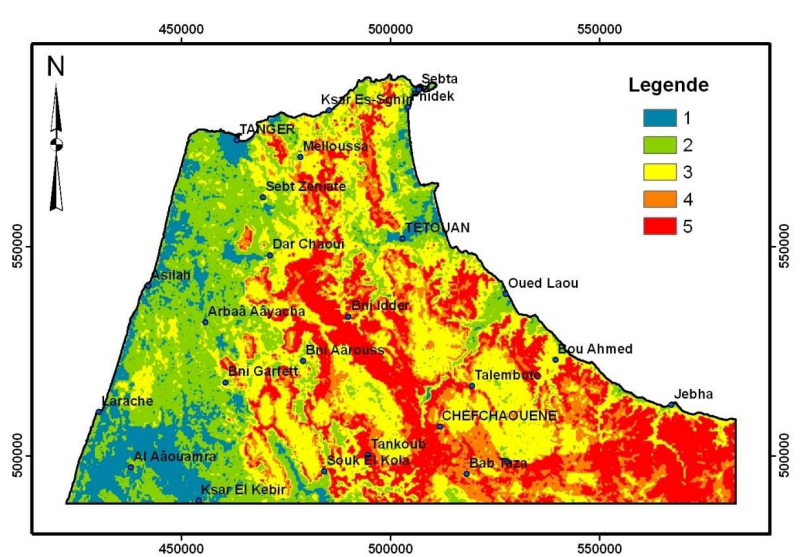

Fig. 10. Carte de la susceptibilité aux instabilités de terrain

La carte de susceptibilité de glissement de terrain obtenue par la méthode AHP montre 5 classes: très faible, faible, moyen, fort et très fort. Elle a été croisée avec les mouvements de masse inventoriés pour tester et évaluer sa validité. Les résultats montrent que : 61,09\% des mouvements de masse identifiés sont situés dans des régions à susceptibilité forte à très forte. $20,32 \%$ des mouvements de masse sont situés dans une zone de susceptibilité moyenne et $18,58 \%$ des mouvements de masse appartiennent à des zones de susceptibilité très faible à faible. Ces derniers s'expliquent par l'apparition de glissements de terrain autour des principaux centres urbains de la péninsule de Tanger et le long des voies de communication déjà construites ou en cours de construction. En conclusion, les valeurs obtenues sont très satisfaisantes pour valider la carte de susceptibilité des mouvements de masse élaborée et le modèle AHP utilisé. La carte de susceptibilité au glissement de terrain obtenue fourni des informations utiles et efficaces sur les instabilités actuelles et leur éventuelle évolution dans le futur, en accord avec l'évolution morphologique de la région. Ce document constitue une contribution à la gestion et à la planification territoriale urbaine et régionale dans le cadre du programme national du développement de la péninsule de Tanger. 


\section{Références}

1. A. M. Lacroix, L'instabilité des versants dans le domaine rifain. Revue Géomorphologie Dynamique. Vol. XV, $\mathrm{n}^{\circ}$ 7, 8, 9, p. 97-109 (1965).

2. G. Maurer. Les montagnes du Rif central, étude géomorphologique. Thèse d'état, Université de Paris, $500 \mathrm{p}$ (1968).

3. A. El Gharbaoui. La terre et l'Homme dans la péninsule tingitane. Etude sur l'Homme et le milieu dans le Rif occidental. Travaux de l'Institut Scientifique, série Géologie et Géographie Physique, Rabat, ${ }^{\circ} 15,439$ p (1981).

4. Y. El Kharim. Etude des mouvements de versants dans la région de Tétouan (Rif occidental): Inventaire, analyse et cartographie. Thèse de Doctorat Es-Sciences, Université Abdelmalek Essaadi, Tétouan, Maroc, 250 p (2002).

5. L. Ait Brahim, M. Mansour, F. Sossey Alaoui et I. El Hammdouni. Rôle de la fracturation dans la désagrégation mécanique des calcaires et la mise en mouvement de la coulée de pierres d'Amtrasse (Rif, Maroc). Edition ISBN 9954-8407-0-2 "L'implication de la géotechnique dans le développement des infrastructures en Afrique ", pp 69-79 (2003).

6. F. Sossey Alaoui. Traitement et intégration des données satellitaires optiques et Radar dans un SIG en vue de l'obtention de carte de l'aléa lié aux instabilités de terrain dans la péninsule de Tanger (Rif septentrional, Maroc). Doctorat, Université Mohamed V, Faculté des Sciences, Rabat. 175p (2005).

7. M. Mansour et L. Ait Brahim. Utilisation de la télédétection pour l'analyse de la fracturation du domaine interne rifain (Maroc): relation avec la répartition des sources. Télédétection, vol. 5, p. 1-2 (2005).

8. M. Mastere, B. Van Vliet Lanoë \& L. Aït Brahim. "Land use mapping and its relation to mass wasting and gullying in NorthWestern Rif (Morocco)." Geomorpholog, relief, process, environment, 3, 335-352, (2013).

9. A. Carrara, E. Catalano, M. Sorriso-Valvo, et al. Digital terrain analysis for land evaluation. Geologia Applicata e Idrogeologia, 1978, vol. 13, p. 69127.Voogd, (1983)

10. M.J. Selby. Hillslope Materials and Processes. Oxford Univ. Press, Oxford (1993).

11. R. Dikau, A. Cavallin, et S. Jäger. Databases and GIS for landslide research in Europe. Geomorphology, vol. 15, no 3-4, p. 227-239 (1996).

12. A.K. Saha, R.P. Gupta, et M.K. Arora. GIS-based landslide hazard zonation in the Bhagirathi (Ganga) valley, Himalayas. International journal of remote sensing, vol. 23, no 2, p. 357-369 (2002).

13. M. Kabbaj, M. Himmi, J. Benbouziane, S. Leroueil, A. Maatouk. Talus du Rif (Analyse générale du phénomène des glissements de terrains au nord du Maroc. L'ampleur, les causes et les remèdes pour le réseau routier). Rapport définitif (Coopération maroco- canadienne). Free Pub. Casablanca, 257p (1995).

14. L. Ait Brahim. Tectoniques cassantes et états des contraintes récents au Nord du Maroc. Contribution al'étude du risque sismotectonique. Thèse Doctorat d'Etat, Université Mohammed V, Rabat, (1991).

15. L. Ait Brahim, et F. Sossey Alaoui. Utilisation de la télédétection pour l'analyse de la fracturation du domaine interne rifain (Maroc): relation avec la répartition des sources. Revue canadienne : Télédétection n22 pp 123-135 (2002).

16. LEE, Saro et TALIB, A. Jasmi. Analyse probabiliste de la susceptibilité aux glissements de terrain et de l'effet des facteurs. Géologie de l'environnement, vol. 47, no 7, p. 982-990 (2005).

17. CL. Bardinet. Méthodes et réalisations en cartographie automatique des données satellitaires. Bulletin de la Société Belge de Géologie, Tomme 97, fascicule 2, p. 101-113 (1988).

18. J.R. Jensen. Introductory digital image processing: a remote sensing perspective. 2nd edition in geographic information science (ed. k. c. clarke), upper saddle river, nj: prentice hall, 316 p (1996).

19. L. Ait Brahim, C. Nakhcha, B. Tadili, A. El Mrabet et N. Jabour. Structural analysis and interpretation of the surface deformations of the feb 24th $2004 \mathrm{Al}$ Hoceima earthquake. Newsletter $\mathrm{N}^{\circ} 21$, Avril, CSEM-EMSC, $\mathrm{N}^{\circ}$ ISSN: 1607-1980, pp 10-12 (2004).

20. A. Tahayt, T. Mourabit, A. Rigo, et al. Mouvements actuels des blocs tectoniques dans l'arc Bético-Rifain à partir des mesures GPS entre 1999 et 2005. Comptes Rendus Geoscience , vol. 340, no 6, p. 400-413 (2008).

21. H. Voogd. Evaluation multicritère pour la planification urbaine et régionale. Londres: Pion, (1983).

22. L. AIT Brahim. Cadre sismotectonique et risque sismique de la chaîne rifaine, Travaux de l'Institut Scientifique, série géographique physique et géologie, $\mathrm{n}^{\circ} 397$ pp.121-135. (2002).

23. B. Deffontaines, $\mathrm{P}$ Chotin, L. Ait Brahim, M. Rozanov. Investigation of active faults in Morocco using morphonetic methods and pattern analysis. Sonderdruck an Geologische Rundschau, Band 81, Helt 1, Stuttgart, pp. 119-210 Allemagne (1992).

24. P. Chotin, L. Ait Brahim, B. Deffontaines, J.P. Rudant J.P, A. Chaouni. Nouvelles données ERS1SAR sur la reconnaissance du réseau de failles dans la péninsule de Tanger Maroc : Télédétection- photo interprétation $\mathrm{n}^{\circ} 1995 / 2, \quad$ p. p129-152, Editions ESKA,France (1995). 